\title{
Serum Levels of miR-155, miR-326, and miR-133b as Early Diagnostic Biomarkers for the Detection of Human Acute Heart Allograft Rejection in Comparison with Serum Cardiac Troponin T
}

\author{
Aghil Esmaeili-bandboni, PhD, ${ }^{1}$ Jamshid Bagheri, $M D,{ }^{2}$ Ali Reza Bakhshandeh, $M D,{ }^{3}$ \\ Javad Mohammadnejad, $\mathrm{PhD},{ }^{4}$ Esmaeil Sadroddiny, $\mathrm{PhD}^{1}$
}

Departments of ${ }^{1}$ Medical Biotechnology, School of Advanced Technologies in Medicine; ${ }^{2}$ Cardiovascular Surgery, Tehran Heart Center; ${ }^{3}$ Cardiovascular Surgery, Imam Khomeini Hospital Complex, Tehran University of Medical Sciences, Tehran, Iran; and ${ }^{4}$ Department of Life Science Engineering, Faculty of New Sciences and Technologies, University of Tehran, Tehran, Iran

\section{ABSTRACT}

Background: Acute heart allograft rejection occurs as a result of antibody-mediated rejection that presents during the first month after transplantation. Finding a non-invasive biomarker is essential for diagnosis of heart allograft rejection. In this research, we intended to compare expression levels of several microRNAs across cardiac troponin T levels between rejected patients (who died before one month following transplantation), non-rejected patients (who survived for at least one month after transplantation), and non-transplanted patients (CABG surgery patients).

Methods: Serum levels of miR-155, miR-326, and miR$133 \mathrm{~b}$ were evaluated by the q-RT-PCR method. Furthermore, cardiac troponin $\mathrm{T}$ levels were measured by a highly sensitive electrochemiluminescence assay. Finally, the data were analyzed by independent sample t-test using SPSS $21^{\circledR}$ computer software.

Results: It was observed that miR-326 and miR-155 expression levels increased after $24 \mathrm{~h}$ and $72 \mathrm{~h}$ of surgery in rejected patients compared with the two other groups, but these increases were not statistically significant. Moreover, the decrease in miR-133b expression level was non-significant after transplantation in the rejected group compared with the non-rejected group. However, cTnT levels in rejected patients increased significantly compared with the other groups $(P<.05)$. After ROC curve analysis, the cTnT marker with the most area under the curve (AUC $=1.00,95 \%$ confidence interval, 1.00 to $1.00 ; P=.006)$, had the best discriminatory power, and among microRNAs, miR-326 had the largest area under curve $(\mathrm{AUC}=0.81)$, and consequently the highest discriminatory power.

Conclusions: We demonstrated that troponin $\mathrm{T}$ can be a more efficient biomarker than miRNAs for early prediction of human death caused by acute heart rejection, and the ROC curves analysis verified this finding.

Received August 10, 2017; received in revised form fanuary 3, 2018; accepted Fanuary 16, 2018.

Correspondence: Esmaeil Sadroddiny, PhD, Associate Professor of Department of Medical Biotechnology, School of Advanced Technologies in Medicine, Tebran University of Medical Sciences, Tebran, Iran; tel: +982143052120, fax: +982188779917 (e-mail:sadroddiny@sina.tums.ac.ir).

\section{INTRODUCTION}

Candidates for allograft transplantation may possess circulating antibodies against human and non-human leukocyte antigens, called allosensitization [Al-Mohaissen 2014]. An allograft transplant can stimulate both cellular and humoral immune responses [Racusen 2003; Rodriguez 2005]. Notwithstanding that immunosuppressive drugs can control the cellular immune response, antibody-mediated rejection (AMR) is a type of immunopathologic process in which graft injury occurs by activated complement. This process does not respond to conventional therapy [Billingham 1990; Rodriguez 2005] and necessitates novel treatments that can reduce anti-HLA antibodies [Djamali 2014].

Acute allograft rejection arises as a result of antibodymediated rejection that presents during the first month after allograft transplantation [Taylor 2000; Michaels 2003; Reed 2006]. The endomyocardial biopsy (EMB) is currently the gold standard for detection of heart allograft rejection [Gradek 2001; Van Huyen 2014]. EMB is an invasive method and expensive for Iranian patients. Therefore, finding a nonor less-invasive biomarker for the diagnosis of heart allograft rejection is essential. Several less invasive biomarkers involved in the detection of heart allograft rejection have been investigated in previous studies, including prolactin [Carrier 1986], cardiac troponin T [Alexis 1998], cardiac troponin I [Siaplaouras 2003], and procalcitonin [Hammer 1998]. Moreover, the up-regulation and down-regulation of microRNAs as biomarkers for early detection of heart allograft rejection have been demonstrated in several studies [Van Huyen 2014].

The use of cardiac troponin- $\mathrm{T}$ (cTnT) as a biomarker for detection of heart transplantation rejection has been studied since 1993 [Zimmermann 1993]; the cTnT level increases in many types of heart disease. With the advent of a highly-sensitive approach such as chemiluminescence, this biomarker was again given attention for use in monitoring heart rejection, and other types of heart disease [Reichlin 2009; Giannitsis 2010; Muñoz-Esparza 2011; Patel 2014].

MicroRNAs are a large part of non-coding RNAs group that plays a critical role in the expression of many genes in eukaryotes [Krol 2010; Ling 2013]. The BIC gene is transcripted and processed for making miR-155, which is responsible for the normal functioning of the immune cells (B cells, $T$ cells, and dendritic cells) and is overexpressed by activation 
Table 1: Patients Characteristics

\begin{tabular}{|c|c|c|c|}
\hline Characteristics & Rejected Pts. & $\begin{array}{c}\text { Non-rejected } \\
\text { Pts. }\end{array}$ & $\begin{array}{c}\text { Non-transplanted } \\
\text { Pts. }\end{array}$ \\
\hline Male/Female $(n / n)$ & $2 / 1$ & $4 / 2$ & $2 / 1$ \\
\hline Age (years) & $29 \pm 5.56$ & $46.5 \pm 9.31$ & $60.33 \pm 11.84$ \\
\hline Weight (kg) & $76.66 \pm 9.71$ & $80.16 \pm 14.55$ & $71.66 \pm 12.50$ \\
\hline $\begin{array}{l}\text { Echocardiography } \\
\text { (EF) value before } \\
\text { surgery }\end{array}$ & $16.66 \pm 2.88$ & $16.66 \pm 2.58$ & $43.33 \pm 2.88$ \\
\hline
\end{tabular}

Genders are based on number; other characteristics are presented as mean $\pm \mathrm{SD}$

of these cells [Trotta 2012]. However, miR-155 expression has a key role in the T-cell-mediated antibody response, adaptive immunity, and the induction of expression of itself by T-cell receptor stimulation [Wilflingseder 2013]. Also, mir-133b, and mir-326 can inhibit anti-apoptotic genes and induce apoptosis in cells [Haas 2011; Patron 2012; Yu 2015]. The increase of these miRNAs can be the cause of antibody mediated rejections, and it has been observed that the level of miR-133b is elevated in heart transplant patients [Wang 2013a].

This study aimed to compare potency of three miRNAs across troponin $\mathrm{T}$ potency in early detection of acute heart allograft rejected in humans. In addition, the specificity of these biomarkers for heart rejection was investigated by comparing rejection patients with CABG surgery patients. The three microRNAs (miR-155, miR-326, miR-133b) expression and troponin $\mathrm{T}$ levels in patients were calculated and analyzed.

\section{MATERIALS AND METHODS}

\section{Study design}

This study was approved by the research committee of Tehran University of Medical Sciences and developed in partnership with Imam Khomeini hospital. A panel reactive antibody test was performed for each patient for selecting the relevant donor. During a one-year period, we collected blood samples from three patients before and after heart transplantation who died one month or less after transplantation (due to acute rejection), and from six patients in the rejected group who survived more than one month following transplantation. All patients underwent similar immunosuppressive therapy, consisting of cyclosporine, mycophenolate mofetil, and prednisolone. Also, for surveying specificity of the rejection biomarkers, blood samples from a group with three patients that were candidates for coronary artery bypass surgery (CABG) were collected. The blood from these patients were collected at various times: before surgery, after 24 hours of surgery (day 1), and after 72 hours of surgery (day 3). All blood samples were centrifuged at 5000 RPM for 7 min, and the supernatants (sera) were stored in the cryotubes at $-80^{\circ} \mathrm{C}$. Some patient characteristics are shown in Table 1 .

\section{miRNA extraction}

Total small RNAs (including miRNAs) were extracted from $250 \mu \mathrm{L}$ sera of patients by GeneAll Hybrid-RTM miRNA purification kits (manufactured in Seoul, South Korea. Cat No: 325-150) according to kit instructions. Subsequently, the concentration and purity of extracted small RNAs were evaluated by NanoDrop One UV-Vis Spectrophotometer (Thermo Fisher Scientific).

\section{Polyadenylation of miRNAs}

Poly A was added to 3' end of $50 \mathrm{ng}$ of purified miRNAs by E. coli Poly(A) Polymerase (purchased from New England BioLabs, Ipswich, Massachusetts, the United States. Cat No: M0276S). This step was done in a Applied Biosystems ${ }^{\mathrm{TM}}$ Veriti 96 Well Thermal Cycler system.

\section{Primers}

Reverse Transcription (RT) primer. The first strand of cDNA was synthesized from miRNA templates using poly- $T$ adaptor cDNA synthesis primer, as described by Shi et al (Shi et al, 2012). Consequently, RT primer for cDNA synthesis was obtained from this article (Shi et al, 2012) and ordered for synthesis in Bioneer company (Daejeon, Seoul, South Korea). The sequence of poly $T$ adaptor primer is: GCGAGCACAG AATTAATACGACTCACTATAGG(T)12VN

\section{$\mathrm{V}=\mathrm{G}, \mathrm{C}, \mathrm{A} ; \mathrm{N}=\mathrm{G}, \mathrm{A}, \mathrm{C}, \mathrm{T}$.}

Reverse Primer. Universal poly(T) adaptor reverse primer was obtained from an article by Shi et al [Shi 2012], and ordered for synthesis by Bioneer (Daejeon, Seoul, South Korea). The sequence of reverse primer is: GCGAGCACAGAATTAATACGAC, $\mathrm{T}_{\mathrm{m}}=57.90^{\circ} \mathrm{C}$.

Forward primers. Forward primers for each miRNA were designed according to the Shi et al and Busk et al protocols [Shi 2012; Busk, 2014], and ordered for synthesis by Bioneer (Daejeon, Seoul, South Korea).

\section{CDNA synthesis}

The first strands of cDNAs were synthesized from total small RNAs using GeneAll HyperScript ${ }^{\mathrm{TM}}$ Reverse Transcriptase kit (Seoul, South Korea, Pd. No: 3033528).

\section{Real-time PCR}

For amplification of miRNAs, the Corbett Rotor-Gene 3000 system was used. The relative expression of miRNAs was analyzed by Pfaffl method (Pfaffl, 2001) according to the following formula:

$$
\text { ratio }=\frac{\left(E_{\text {target }}\right)^{\Delta C P_{\text {target }}(\text { control-sample })}}{\left(E_{\text {ref }}\right)^{\Delta C P_{\text {ref }}(\text { control-sample })}}
$$

where $\mathrm{E}_{\text {target }}$ is efficiency of target primers (miR-155, miR$326, \mathrm{miR}-133 \mathrm{~b}$ ), $\mathrm{E}_{\text {ref }}$ is efficiency of reference primer (miR16), $\Delta \mathrm{CP}$ is the change in crossing point, $E^{\mathrm{w}}$ hiqug is the the as $\Delta \mathrm{CT}$.

In this study, each patient's post-surgery data were analyzed and compared to data obtained before surgery. Then, each patient's pre- surgical data were used as the control group. The relative expression of miRNAs in serum was normalized 
to the expression of miR-16-5P.

For calculating E, a standard curve of microRNAs amplification by its own primers was drawn. Serial dilutions of cDNA provided and standard curves were sketched by Origin 6.0 portable software. E was calculated by

\section{Measurement of Serum cTnT}

The serum levels of cTnT were measured by the electrochemiluminescence technique. The equipment used for this method was Elecsys 2010 analyzer (Roche and Hitachi).

\section{Statistical analysis}

Statistical analysis was done by SPSS $^{\circledR}$ version 21 computer software. An independent sample t-test was used for the comparison of miRNAs expression between rejected patients (dead) and non-rejected patients. Also, for comparing rejected patients (dead) versus non-transplanted groups, an independent sample t-test was used. All $P$-values $<.05$ were considered statistically significant. The area under curves (AUCs) was assessed to determine discriminatory power of each marker. All graphs were drawn by GraphPad Prism 6.0 and Origin 9.1 computer software.
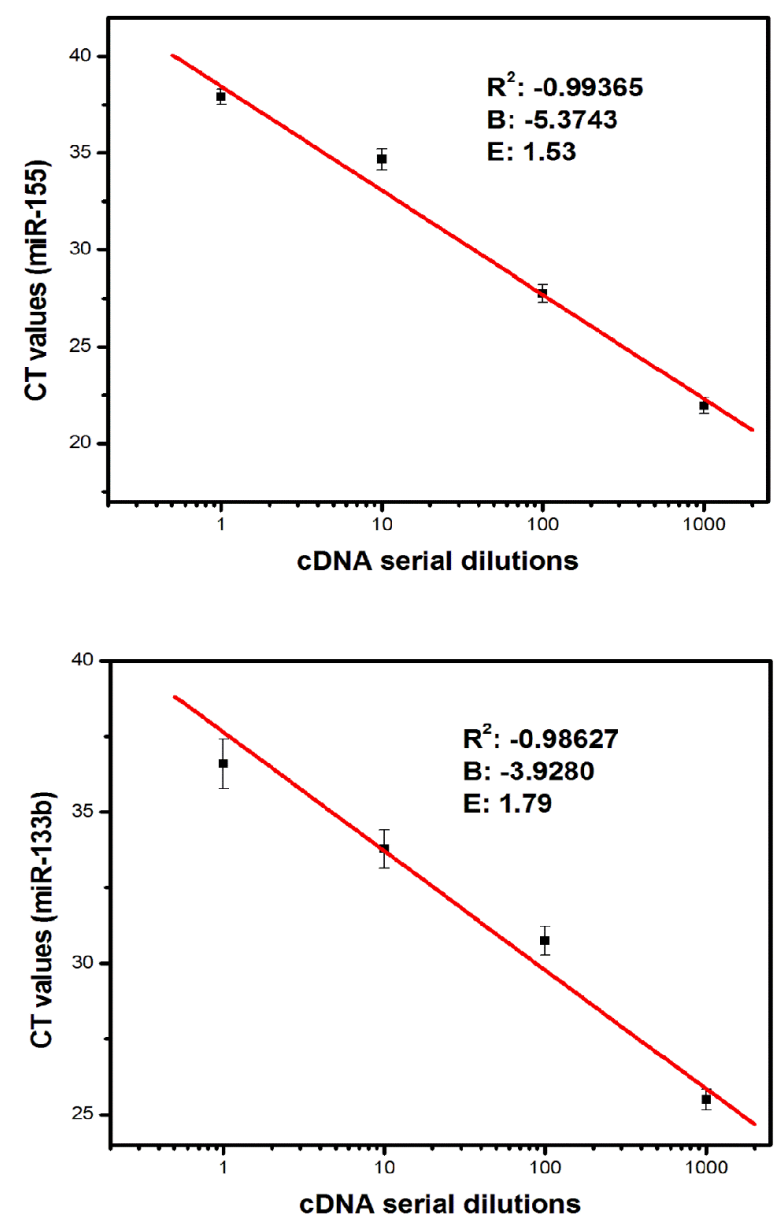

\section{RESULTS}

\section{Standard curves of microRNAs amplification and efficiency} calculation of $q R T-P C R$

Standard curves were drawn in order to verify the accuracy of primers and to calculate the efficiency (E) of real-time PCR. As observed, for all of four miRNAs, R2 values were $\geq$ 0.98 indicating a high accuracy of primers in real-time PCR assay. Slope and $\mathrm{E}$ values for each primer are shown on the graph (Figure 1).

Non-significant up-regulation of miR-326 and miR-155 levels

After 24 hours of surgery, it was observed that miR-155 and miR-326 expression levels increased in the rejected group compared with the non-rejected group. After statistical analysis by unpaired two-tailed T-test, no significant up-regulation was observed, $P=.16$ for miR-155 and miR326. In addition, to compare mean fold changes of expression levels of miR-155 and miR-326 at 24h, between the rejected group and the non-transplanted group, it was shown that there was an increased level of expression in the rejected group compared with the non-transplanted group, which was
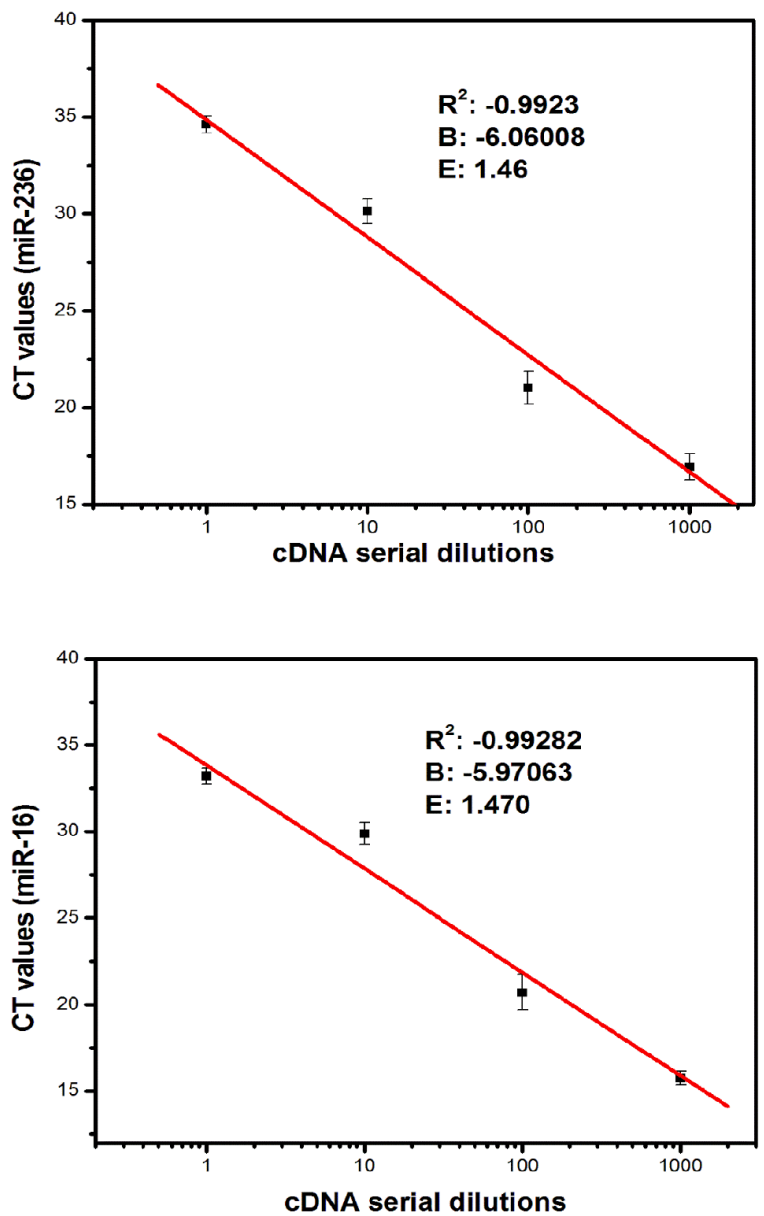

Figure 1: Standard curves of miRNAs amplification using four primers. R2 value is $>0.98$ depicting the accuracy of primers for amplification. B and E represent slope and efficiency, respectively. 

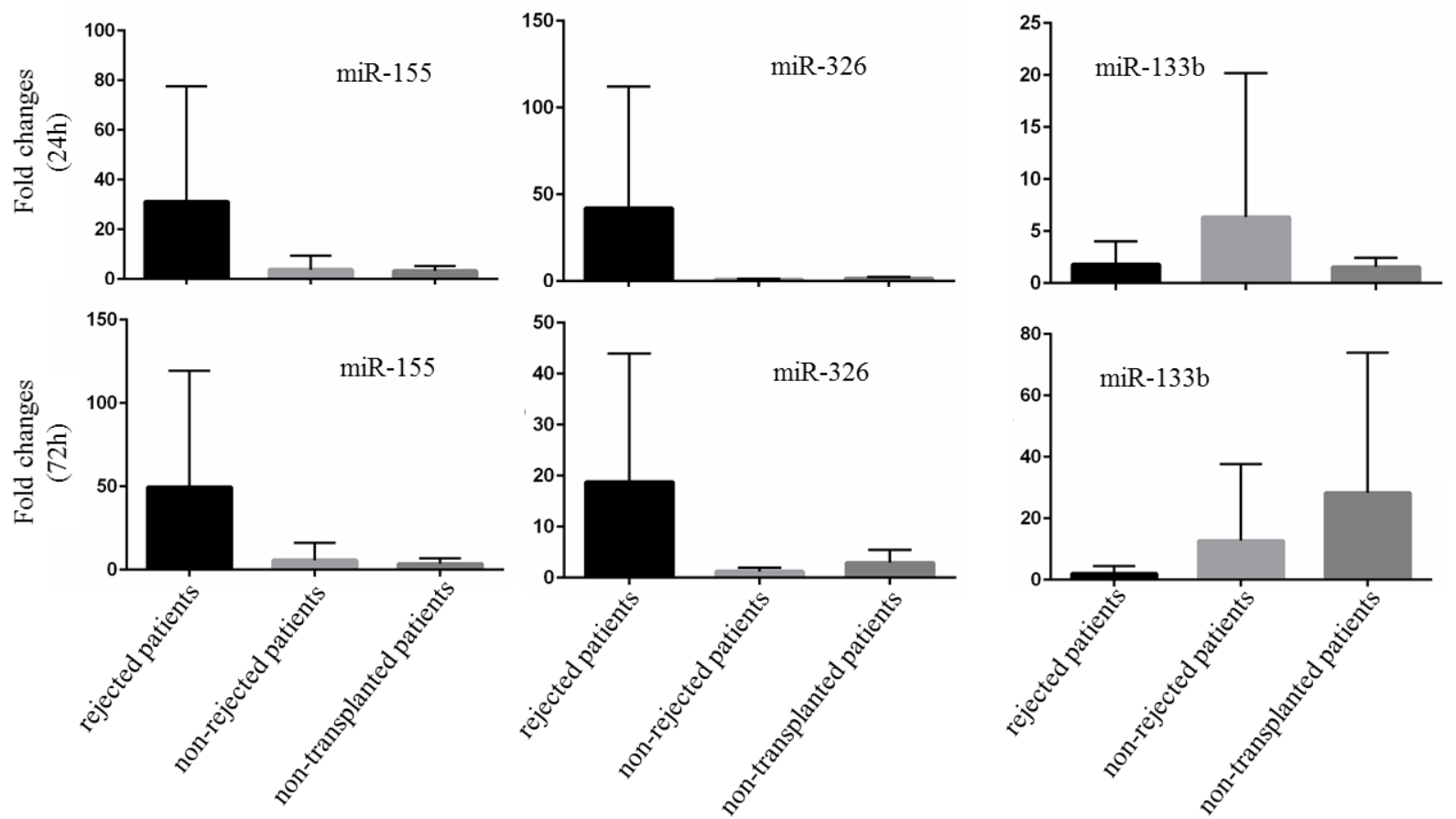

Figure 2: Relative expression of miR-155, miR-326, miR-133b after $24 \mathrm{~h}$ and $72 \mathrm{~h}$ of surgery; $\mathrm{Y}$ axis represents fold changes relative to before surgery using the Pfaffl method. As observed, after 24h of surgery expression levels of miR-155, and miR-326 increased in rejected patients versus non-rejected and non-transplanted patients (top panel). Also, after $72 \mathrm{~h}$ of surgery, a similar trend was observed (bottom panel). The findings have shown these biomarkers have specificity for rejected groups, and their increase $24 \mathrm{~h}$ and $72 \mathrm{~h}$ after transplantation may be a bad prognostic sign for rejection. The changes between groups in expression levels of miR-133b at $24 \mathrm{~h}$ after surgery were not regular (top panel). But after $72 \mathrm{~h}$, the levels decreased in rejected patients versus non-rejected and non-transplanted patients (bottom panel); this decrease may be a bad prognostic sign for rejection. However, all of these finding were not significant $(P>.05)$.

statistically not significant, $P=.35$ for miR-155 and miR326 , respectively. After $72 \mathrm{~h}$ of surgery, comparing the mean expression levels of miR-155 and miR-326, we observed high levels of miR-155 in the rejected group compared to the non-rejected group. This increase was not significant, $P=.12$ and $P=.08$ for miR-155 and miR-326, respectively. Conversely, there was no significant up-regulation between the rejected group and non-transplanted group, $P=.30$ and $P$ $=.32$ for miR-155, and miR-326 respectively (Figure 2).

For miR-133b after $24 \mathrm{~h}$ of surgery, the mean fold changes and statistical analysis by unpaired two-tailed t-tests showed that the rejected patients had lower levels of expression compared to the non-rejected patients, which was not significantly different. However, there was no change between the rejected group and the non-transplanted group. Conversely, the mean fold changes of miR-133b after $72 \mathrm{~h}$ of surgery in the rejected group was lower but not statistically significant compared with the non-rejected group, and a similar trend was observed for the non-transplanted group $P=.58$ and $P=$ .49 respectively (Figure 2 ).

\section{Serum cTnT levels increased in the rejected patients}

A significant increase was observed for mean cTnT levels in the rejected group versus the non-rejected group, for the two times studied, $P=.0001$ and $P=.02$ respectively. There was a significant difference observed between the rejected group and non-transplanted group at both times, $P=.0003$ and $P=.04$ respectively (Figure 3 ).

\section{High discriminatory power of cTnT marker}

To evaluate the discriminatory power of the three miRNAs and $\mathrm{c}_{\mathrm{n}} \mathrm{T}$ biomarkers for heart rejection, receiver operating characteristic (ROC) curve analysis for all patients between each of two comparable groups (between the rejected patients and non-rejected patients, and between the rejected patients and non-transplanted patients) were performed. As shown in Figure 4, cTnT had the best discriminatory power. For this marker, AUC in comparison between the rejected patients and non-rejected patients was 0.98 (95\% confidence interval $[\mathrm{CI}], 0.93$ to $1.00 ; P=.002$ ) and AUC in comparison between the rejected patients and non-transplanted patients was 1.00 ( $95 \%$ confidence interval $[\mathrm{CI}], 1.00$ to $1.00 ; P=$ .006). However, the miRNAs discriminatory power was very low, probably due to a low number of samples analyzed in this study. However, among microRNAs, miR-326 had the highest level of AUC (which in the comparison between the rejected patients and non-rejected patients was 0.81 with $95 \%$ confidence interval $[\mathrm{CI}], 0.59$ to $1.00 ; P=.04$ ) and consequently the highest discriminatory power. The discriminatory power of markers between the rejected patients and 

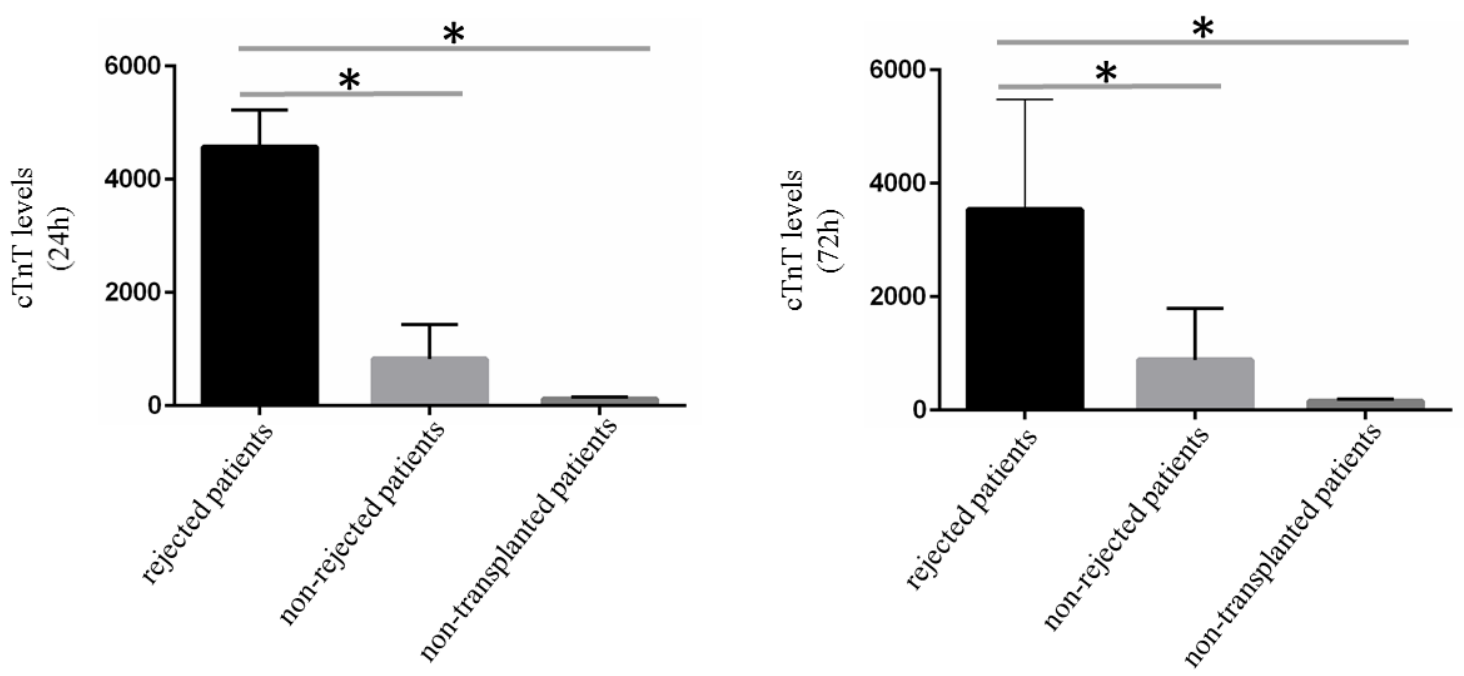

Figure 3: Increase in cTnT levels after $24 \mathrm{~h}$ and $72 \mathrm{~h}$ of surgery; As observed, there was significant differences between the rejected group as compared to the two other groups (non-rejected group, and non-transplanted group) $24 \mathrm{~h}$ (left panel) and $72 \mathrm{~h}$ after surgery (right panel). *depicts $P<.05$.

non-rejected patients (Figure 4A), and between the rejected patients and non-transplanted patients (Figure 4B) were evaluated separately.

\section{DISCUSSION}

Heart allograft transplantation is an ultimate strategy for treating advanced heart failure patients. However, after transplantation, the possibility of rejection of a transplanted graft is a major concern, especially acute rejection that increases the probability of death during the first month after transplantation. Although finding an approach for early detection of rejection has been a challenging issue, an important criterion for an adequate approach is that it be non-invasive. In this research, we used blood samples that are less invasive than the EMB method. This research is one of the few studies on the molecular diagnostics of heart transplantation rejection in Iran.

Van Huyen et al demonstrated that four miRNAs (miR10a, miR-155, miR-31, and miR-92a) increased significantly in patients with rejection of heart allograft transplants [Van Huyen 2014]. The difference between this research and the present study is that in the Van Huyen study, all transplanted patients were divided into two main groups based on EMB results, including the normal group (without any symptom of heart rejection) and rejected groups ( $\mathrm{T}$ cell mediated rejection (TCMR) and antibody mediated rejection (ABMR) with different symptoms of rejection). In our study, because of the loss of EMB results, this grouping changed to the rejected group (patients that died before one month after transplantation because of acute heart rejection) and non-rejected group (patients that survived for at least one month after transplantation). EMB is an invasive clinical tool, and clinical laboratory techniques are very expensive in Iran and insurance companies do not cover it. In addition, surgeons stated that they were challenged by interpretation of $\mathrm{EMB}$ results. For these reasons, $\mathrm{EMB}$ was removed from the list of assays after transplantation in Imam Khomeini hospital. Other differences were the number of patients in each of the groups; in our study, the number of patients was very small, but Van Huyen's study collected at least 30 sample in 5 years. We had expected that with the small number of samples, there would be no significant results. This prediction was correct for miRNAs markers, but for cTnT, despite the small sample size, we did get significant results.

In many of previous transplantation studies we observed the use of $\Delta \Delta \mathrm{CT}$ method, which was the $\Delta \mathrm{CT}$ of miRNA in a patient - mean $\Delta C T$ of population miRNA [Wei 2012; Van Huyen 2014]. But, in this study, we analyzed the real time PCR raw data by comparing the amount of miRNA 24h or $72 \mathrm{~h}$ after surgery to that before transplantation. By that, we evaluated the fold changes after transplantation per patient relative to the amount of miRNA in the same patient before transplantation. Due to the effect of age, gender and body mass index on miRNAs levels [Simon 2014; Kwekel 2015] each of patient must be compared with him/herself. Furthermore, we used the Pfaffl method rather than $\Delta \Delta C T$ method. This method is based on the efficiency of real-time PCR by each primer and has a higher accuracy than $\Delta \Delta \mathrm{CT}$ method since there were different primer efficiencies for various miRNAs [Pfaffl 2004].

In this study, we found that miR-155 and miR-326 are increased 24 hours after surgery in the rejected group compared to the non-rejected group, or non-transplanted group. The increase in these miRNA levels was also seen in previous studies [Dewi 2013; Van Huyen 2014]. Increased expression level of miRNAs in the rejected group compared to the nontransplanted group shows that these markers had specificity for transplantation and did not increase in other heart surgery patients (CABG). We found that miR-133b decreased after transplantation, unlike other previous studies [Wang 2013b]. But these results were not statistically different. These statistical results are as a result of limited time, giving rise to small 
A
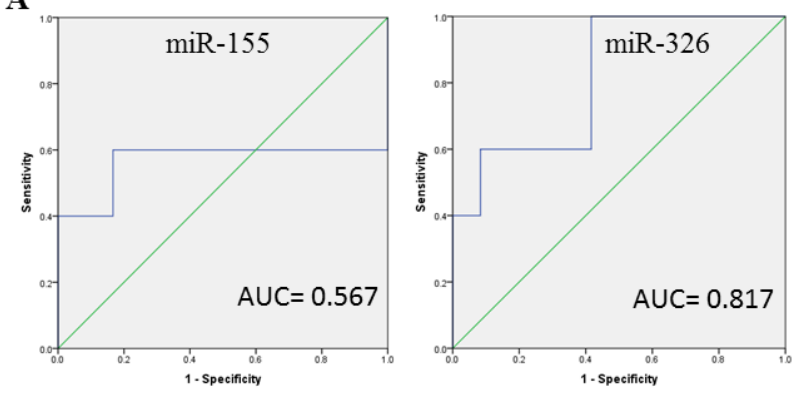

B
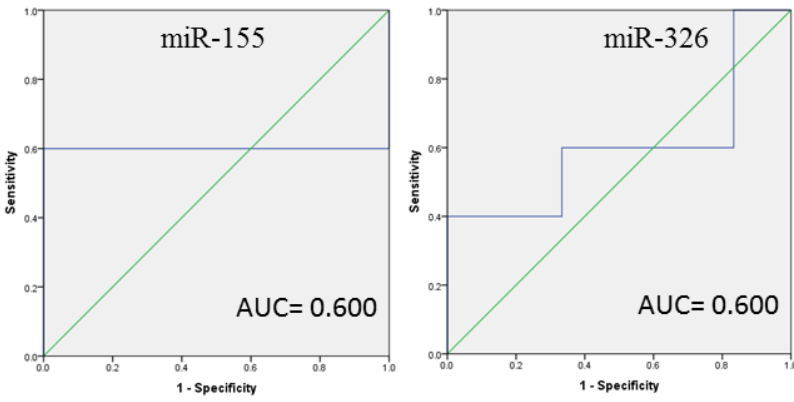
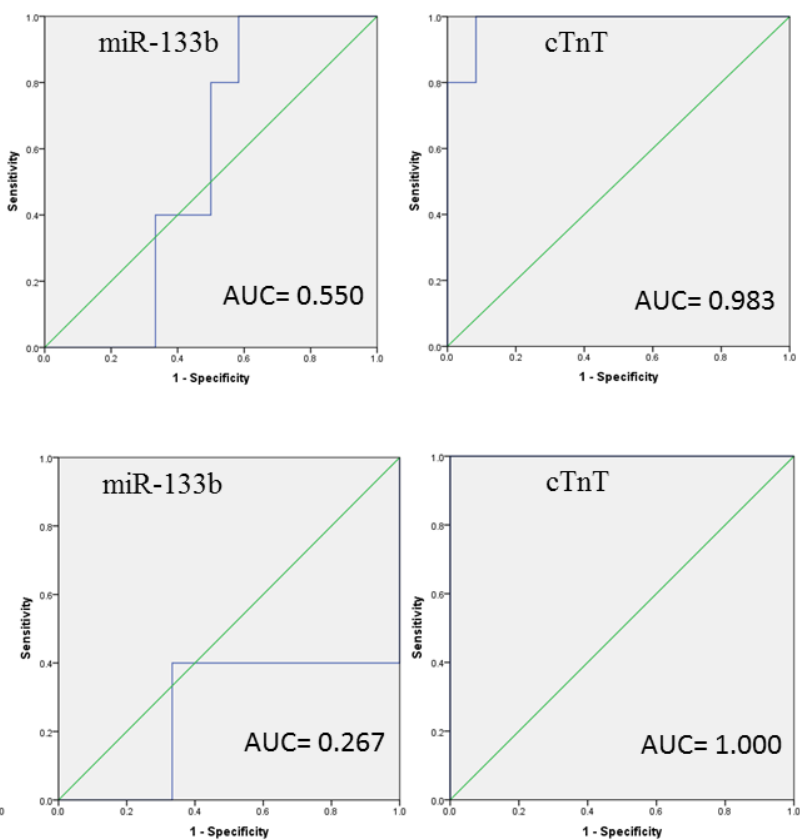

Figure 4: ROC curves analysis of miRNAs and cTnT based on; (A) comparing rejected patients and non-rejected patients, (B) comparison between rejected patients and non-transplanted patients. AUC depict the "area under curve". As observed AUC for the cTnT marker is higher than three miRNAs markers in both types of comparisons.

sampling number. Troponin T highly sensitive detection demonstrated that its level was significantly increased in the rejected patients as compared to the non-rejected patients, and non-transplanted patients. Therefore, we demonstrated that troponin $\mathrm{T}$ can be a better biomarker for early detection of human death caused by acute heart rejection than miRNAs. In addition, ROC curve analysis verified these findings. With ROC curve analysis we found microRNAs biomarkers can not be used a suitable markers for the prognosis of rejected patients versus non-rejected patients.

There are several points which might have contributed to this result. First, the small sample size of patients might possibly decrease the power of microRNAs to discriminate between rejected patients and non-rejected patients, and between rejected patients and non-transplanted patients. In the other words, in small size conditions ROC curve analysis may not be reliable [Hanczar 2010]. However, ROC curve analysis for the cTnT marker showed that it had an AUC closer to 1.00 and appropriate discriminatory power, even in a small sample size. It verifies the previous finding by $\mathrm{Li}$ et al that $\mathrm{c} T \mathrm{n} \mathrm{T}$ discriminatory power was better than microRNAs power in the detection of acute myocardial infarction [Li 2013]. Second, a power technique such as the high-sensitive chemiluminescence was used for the cTnT, while the poly-adenylation method for microRNAs amplification is not highly sensitive, but, because it is the most common and inexpensive technique compared to the Taq-Man based amplification, we employed it in this study. As previously mentioned, this research is one of the few studies on the molecular diagnostics of heart transplantation rejection in Iran. We think that with a larger sample size and the use of highly-sensitive methods, such as microarray or Taq-Man based amplification, microRNAs may prove to be more powerful markers than cTnT.

\section{CONCLUSION}

Our study shows that detection of serum levels of cTnT by chemiluminescence is more efficient than the detection of serum levels of miRNAs by qPCR based on polyadenylation, for diagnosis of human heart allograft rejection.

\section{ACKNOWLEDGEMENTS}

We thank all the nurses of Imam Khomeini Hospital that helped us in collecting the blood samples. We also appreciate Tehran Heart Center Laboratory experts for their assistance with high-sensitive electrochemiluminescence.

\section{REFERENCES}

Al-Mohaissen M A and Virani S A. 2014. Allosensitization in heart transplantation: an overview. Can J Cardiol 30:161-172.

Alexis J D, Lao C D, Selter J G, et al. 1998. Cardiac troponin T: a noninvasive marker for heart transplant rejection? J Heart Lung Transplant 17:395-398.

Billingham M, Cary N, Hammond M, et al. 1990. A working formulation for the standardization of nomenclature in the diagnosis of heart and lung rejection: Heart Rejection Study Group. The International Society for Heart Transplantation. J Heart Transplant 9:587-593. 
Busk P K. 2014. A tool for design of primers for microRNA-specific quantitative RT-qPCR. BMC Bioinformatics 15:29.

Carrier M, Russell D, Wild J, et al. 1986. Prolactin as a marker of rejection in human heart transplantation. J Heart Transplant 6:290-292.

Dewi I S, Torngren K, Gidlöf O, et al. 2013. Altered serum miRNA profiles during acute rejection after heart transplantation: potential for non-invasive allograft surveillance. J Heart Lung Transplant 32:463-466.

Djamali A, Kaufman D, Ellis T, et al. 2014. Diagnosis and management of antibody-mediated rejection: Current status and novel approaches. Am J Transplantation 14:255-271.

Giannitsis E, Kurz K, Hallermayer K, et al. 2010. Analytical validation of a high-sensitivity cardiac troponin $\mathrm{T}$ assay. Clin Chem 56:254-261.

Gradek W Q, D'Amico C, Smith A L, et al. 2001. Routine surveillance endomyocardial biopsy continues to detect significant rejection late after heart transplantation. J Heart Lung Transplant 20:497-502.

Haas J D, Nistala K, Petermann F, et al. 2011. Expression of miRNAs miR-133b and miR-206 in the Il17a/f locus is co-regulated with IL-17 production in $\alpha \beta$ and $\gamma \delta$ T cells. PLoS One 6:e20171.

Hammer S, Meisner F, Dirschedl P, et al. 1998. Procalcitonin: a new marker for diagnosis of acute rejection and bacterial infection in patients after heart and lung transplantation. Transplant Immunol 6:235-241.

Hanczar B, Hua J, Sima C, et al. 2010. Small-sample precision of ROCrelated estimates. Bioinformatics 26:822-830.

Krol J, Loedige I and Filipowicz W. 2010. The widespread regulation of microRNA biogenesis, function and decay. Nature Reviews Genetics 11:597-610.

Kwekel J C, Vijay V, Desai V G, et al. 2015. Age and sex differences in kidney microRNA expression during the life span of F344 rats. Biol Sex Differ 6:1.

Li Y-Q, Zhang M-F, Wen H-Y, et al. 2013. Comparing the diagnostic values of circulating microRNAs and cardiac troponin $\mathrm{T}$ in patients with acute myocardial infarction. Clinics 68:75-80.

Ling H, Fabbri M and Calin G A. 2013. MicroRNAs and other noncoding RNAs as targets for anticancer drug development. Nature reviews Drug discovery 12:847-865.

Michaels P J, Espejo M L, Kobashigawa J, et al. 2003. Humoral rejection in cardiac transplantation: risk factors, hemodynamic consequences and relationship to transplant coronary artery disease. J Heart Lung Transplant 22:58-69.

Muñoz-Esparza C, Garrido I P, Blanco R, et al. 2011. Usefulness of high sensitivity troponin $\mathrm{T}$ assay in detecting acute allograft rejection after heart transplantation. Revista Española de Cardiología (English Edition) 64:1109-1113.

Patel P C, Hill D A, Ayers C R, et al. 2014. High-Sensitivity Cardiac Troponin I Assay to Screen for Acute Rejection in Patients With Heart Transplant CLINICAL PERSPECTIVE. Circ Heart Fail $7: 463-469$.

Patron J P, Fendler A, Bild M, et al. 2012. MiR-133b targets antiapoptotic genes and enhances death receptor-induced apoptosis. PLoS One 7:e35345.
Pfaffl M W. 2001. A new mathematical model for relative quantification in real-time RT-PCR. Nucleic Acids Res 29:e45-e45.

Pfaffl MW. Quantification strategies in real-time PCR. In: Bustin, Stephen A. 2004. A-Z of quantitative PCR. La Jolla, CA: International University Line. 87:112.

Racusen L C, Colvin R B, Solez K, et al. 2003. Antibody-Mediated Rejection Criteria-an Addition to the Banff' 97 Classification of Renal Allograft Rejection. Am J Transplantation 3:708-714.

Reed E F, Demetris A J, Hammond E, et al. 2006. Acute antibody-mediated rejection of cardiac transplants. J Heart Lung Transplant 25:153-159.

Reichlin T, Hochholzer W, Bassetti S, et al. 2009. Early diagnosis of myocardial infarction with sensitive cardiac troponin assays. New Engl J Med 361:858-867.

Rodriguez E, Skojec D V, Tan C D, et al. 2005. Antibody-Mediated Rejection in Human Cardiac Allografts: Evaluation of Immunoglobulins and Complement Activation Products C4d and C3d as Markers. Am J Transplantation 5:2778-2785.

Shi R, Sun Y-H, Zhang X-H, et al. 2012. Poly (T) Adaptor RT-PCR. Next-Generation MicroRNA Expression Profiling Technology: Methods and Protocols 53-66.

Siaplaouras J, Thul J, Kramer U, et al. 2003. Cardiac troponin I: a marker of acute heart rejection in infant and child heart recipients? Pediatr Transplant 7:43-45.

Simon L M, Edelstein L C, Nagalla S, et al. 2014. Human platelet microRNA-mRNA networks associated with age and gender revealed by integrated plateletomics. Blood 123:e37-e45.

Taylor D O, Yowell R L, Kfoury A G, et al. 2000. Allograft coronary artery disease: clinical correlations with circulating anti-HLA antibodies and the immunohistopathologic pattern of vascular rejection. J Heart Lung Transplant 19:518-521.

Trotta R, Chen L, Ciarlariello D, et al. 2012. miR-155 regulates IFNproduction in natural killer cells. Blood 119:3478-3485.

Van Huyen J-P D, Tible M, Gay A, et al. 2014. MicroRNAs as non-invasive biomarkers of heart transplant rejection. Eur Heart J 35:194-3202.

Wang E, Nie Y, Zhao Q, et al. 2013a. Circulating miRNAs reflect early myocardial injury and recovery after heart transplantation. J Cardiothorac Surg 8:1.

Wang E, Nie Y, Zhao Q, et al. 2013b. Circulating miRNAs reflect early myocardial injury and recovery after heart transplantation. Journal of cardiothoracic surgery 8:165.

Wei L, Wang M, Qu X, et al. 2012. Differential expression of microRNAs during allograft rejection. Am J Transplantation 12:1113-1123.

Wilflingseder J, Regele H, Perco P, et al. 2013. miRNA profiling discriminates types of rejection and injury in human renal allografts. Transplantation 95:835.

Yu S, Huang H, Deng G, et al. 2015. miR-326 targets antiapoptotic Bcl-xL and mediates apoptosis in human platelets. PLoS One 10:e0122784.

Zimmermann R, Baki S, Dengler T, et al. 1993. Troponin T release after heart transplantation. Br Heart J 69:395-398. 